\title{
Next Generation Manufacturing Systems in the IMS Program
}

\author{
T. Kurihara
}

Fuji Electric Co., Ltd

Tokyo 100, Japan

Tel: +81335368825 Fax: +81332010750

kurihara@fujielectric.co.jp

\section{J. Jordan}

Consortium for Advanced Manufacturing - International

3301 Airport Freeway, Suite 324

Bedford, TX 76021 USA

Tel: +1 8178601654 Fax: + 18172756450

jajordan@ix.netcom.com

\section{P. Bunce}

Consortium for Advanced Manufacturing - International

20 Market Street

Poole, Dorset, BH15 INF, UK

Tel: +441202670717 Fax: +441202680698

peter@cam-i.demon.co.uk

\begin{abstract}
The International Intelligent Manufacturing Systems (IMS) Program is an important program of cooperative research projects, one of which is the Next Generation Manufacturing Systems (NGMS) project. NGMS seeks to develop the technologies and methodologies needed for the manufacturing systems that will support the next generation of manufacturing enterprise. NGMS is integrating thinking on advanced manufacturing systems from Europe (the fractal factory), from Japan (autonomous and distributed manufacturing systems, biological manufacturing systems) and the United States (agile manufacturing). We give an overview of requirements for NGMS and a summary of the applied research the project is undertaking.
\end{abstract}




\section{Keywords}

Advanced manufacturing systems, intelligent systems, fractals, agility. Intelligent Manufacturing Systems (IMS) Program, Next Generation Manufacturing Systems (NGMS).

\section{THE INTELLIGENT MANUFACTURING SYSTEMS (IMS) PROGRAM}

The IMS Program was conceived in Japan in 1989 as an international, industry driven program of collaborative research and development (R\&D). After negotiations, the Japanese proposal was accepted on a provisional basis and a set of test cases begun in 1993. After further negotiations, Australia, Canada, Japan, Switzerland and the United States agreed to Terms of Reference (ToR) for a full scale, 10 year program beginning in 1995. (As of June 1996, the European Union is expected to ratify the ToR). In parallel, the Japanese IMS Promotion Centre established a Domestic Japan IMS Program. In September 1995, the IMS Steering Committee endorsed the first full scale projects. The IMS Program is comprehensive, with major technical themes that span the needs of manufacturing enterprises for the early Twenty First Century:

- Total Product Life Cycle Issues, including future general models of manufacturing systems.

- Process Issues, including process technology innovation, more flexible and more autonomous processing modules, and better interaction and harmony among various components and functions.

- Strategy/Planning/Design Tools, including methods and tools for business process re-engineering, to support the analysis and development of manufacturing strategies, and to support planning in an extended enterprise or virtual enterprise environment.

- Human/Organization/Social Issues, including improved capability of manufacturing workforce/education, training, autonomous offshore plants, and corporate technical memory.

- Virtual/Extended Enterprise Issues.

\section{NEXT GENERATION MANUFACTURING ENTERPRISES (NGMES)}

Next Generation Manufacturing Systems (NGMS) will support Next Generation Manufacturing Enterprises (NGMEs). NGMEs have been characterized (Jordan, 1994a) as dynamically combining customers, multiple design and production entities, and suppliers, into organizations that will form to meet a customer need, fulfill the need, and then dissolve. NGMEs are expected be the dominant form of manufacturing enterprise in a time of unpredictable competitive challenges and a rapidly, chaotically, changing global business environment.

The important elements of Next Generation Manufacturing Enterprises are:

- NGMEs will be customer-driven. Customers will be deeply integrated into all aspects of the product cycle.

- Suppliers will be integrated into the product cycle. Sub system suppliers, especially, will become peers.

- As illustrated by much of the thought about NGMEs, e.g. the thinking underlying the Japanese Autonomous and Distributed Manufacturing Systems and Biological 
Manufacturing Systems efforts (IMS93-II-1 Group, 1994, the Fraunhofer Society's Fractal Company (Wamecke, H.-J., 1993), and the U.S. Agile Manufacturing activities (Nagel, R., et.al. 1991), and the view of the factory floor; rigid, static, hierarchical, manufacturing enterprises will be replaced by virtual enterprises exhibiting great adaptability to rapid change and able to produce small lots with high quality and at low costs.

- NGMEs will be made up of simple, distributed, autonomous but cooperating, work units, that will work in flattened, network like, organizations.

- The global economy and the technologies for telecollaboration will both require and enable work units to be distributed globally.

\section{INTRODUCTION TO NGMS}

NGMS will support the product life cycle within NGMEs. That is, NGMS will integrate a dynamically changing collection of self-organizing, autonomous but cooperating, distributed work units executing the processes that relate specifically to products and their development, production, distribution, maintenance, field enhancement, and disposal. These processes will have to be integrated and supported at the Enterprise Level, Factory Level, and the Factory Floor. (Additional systems, integrated at a high level with NGMS, will support the other processes of an NGME.)

Key Characteristics of NGMS

- Support for virtual enterprise

- Customer focused and business centred

- Reconfigurable, adaptable, and flexible in response to customers

- Global support for design and production

- Information and knowledge based, human intelligence oriented

- Modular to support distribution and autonomy, but cooperative to achieve enterprise goals

- Environmentally aware

Key characteristics of NGMS were developed at an NGMS Program Definition Workshop (Jordan, 1994b) conducted in February 1994 for .twenty large manufacturing companies from Europe, Japan, and the United States. The Workshop also identified a longer list of needs that the NGMS should meet.

\section{NGMS ARCHITECTURE}

Four concepts: Agile Manufacturing, the Fractal Company, Bionic Manufacturing Systems (BMS) and Autonomous and Distributed Manufacturing Systems (ADMS) provide the basis for the NGMS architecture. Each of the concepts being developed in different parts of the world, contributes to meeting the requirements of highly adaptive NGMS able to support competitive, agile, manufacturing enterprises. Each assumes that NGMEs will be organized into distributed work units with a high degree of autonomy and intelligent behaviour. The concepts deal with different aspects of manufacturing systems; the R\&D will reach fruition at different times, but the combination of these four views is a powerful, representation of advanced manufacturing systems. 
Needs NGMS Must Meet:

- Be a Global System.

- Be a Portable System.

- Be an Integrated System.

- Be Supportive of Integrated Product/ Process Design (IPPD).

- Support and Use Human Intelligence.

- Support Intelligent Machines.

- Provide "Just-in-Time" Information.

- Be Agile.

- Be Model and Simulation Based for Virtual Manufacturing.

- Be Environmentally Oriented.

- Include Process Control

NGMEs will have fewer levels of hierarchy and will have information systems capable of conveying floor level information throughout the enterprise. Time constraints will make it important for the enterprise to have an accurate understanding of the status of the floor level operations in order to make timely decisions affecting enterprise level activities. Because of this, NGMS will be more tightly integrated across the floor, factory, and enterprise levels. It will be difficult to decompose NGMS in the traditional hierarchies or levels and so it is important to take the best ideas at all levels and bring them together in a unified view of NGMS.

Agility provides a philosophical basis for NGMS. Agility speaks to the capabilities of an enterprise to reconfigure itself quickly in response to sudden changes, but in ways that are timely, cost-effective, of a broad scope, and robust. Agility theory seeks to provide metrics for business processes, for physical operations, and for human resources to respond to rapid and unpredictable change. The emphasis on agility implies that time must be treated very carefully in NGMS models and simulations, and that NGMS must include instrumentation and analysis tools for work unit, factory, and enterprise level measures.

The Fractal Company describes an organization, made up of self similar, self organizing, autonomous work units (fractals). A strength of the Fractal Company concepts is the guidance they give to business process re-engineering, to the propagation of goals, and to the human element in NGMEs. Although work units will have wide latitude about how they accomplish their tasks in the virtual manufacturing enterprise, they will have to align their goals with those of the enterprise. Fractal Company R\&D is building a manual methodology for goal setting and propagation (termed navigation) in enterprises organized into empowered work teams. This methodology appears also to be applicable in more loosely coupled enterprises.

Autonomous Distributed Manufacturing System (ADMS), suggested in Japan, aims to realize the autonomous distribution of modules of manufacturing system, by giving intelligence to each of the modules. Here the manufacturing system is composed of module units, which are functioning autonomously and cooperatively, and are integrated into a virtual production system.

Biological Manufacturing Systems (BMS), which is the further advanced concept of ADMS, have the functions imitating those of biological organisms, such as self organization, self 
recovery, self growth and evolution, and will provide the methodology covering all the levels. Here we intend to realize the manufacturing system which can quickly respond to needs and is harmonious to natural environment, by systematizing the information of a product throughout its whole life cycle, which consists of planning, design, production, consumption and disposal.

In this research we will try to develop decision support systems and architectures which provide common frameworks for manufacturing systems in the course of transition. These will change from the current concentrated manufacturing system to an autonomous distributed manufacturing system, and further on to a biological manufacturing system.

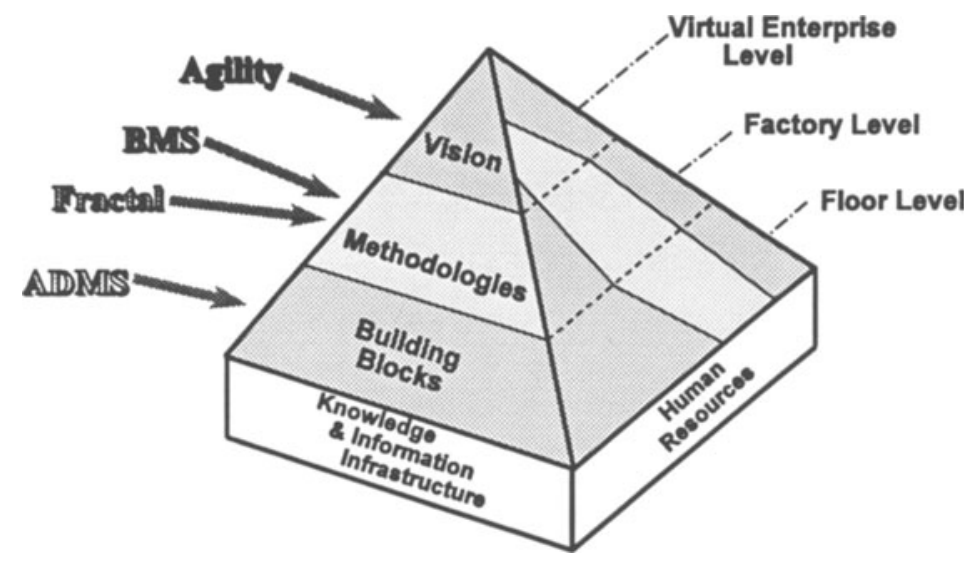

Figure 1

\section{THE NGMS IMS PROJECT:}

\section{DESCRIPTION, MODELLING AND SIMULATION OF NGMS}

In late 1993 an international partnership of leading manufacturing companies, supported by a strong group of research universities, came together to set an R\&D agenda whose results will be the technologies, methodologies, and sub systems needed to transform today's manufacturing systems into the ones that will best support NGMEs.

Building on an excellent base of work in Europe, Japan, and the United States, the partnership developed a comprehensive R\&D agenda that spans the product life cycle and all key issues in manufacturing. Working through the agenda will take a decade.

The first set of tasks on the agenda form the basis for a proposal made by the consortium to the IMS Program, a proposal called Description, Modelling, and Simulation of Next Generation Manufacturing Systems (NGMS): Merging the Agile, Autonomous and Distributed, Biological, and Fractal Manufacturing Systems Concepts. The proposed project was endorsed by the International IMS Steering Committee in September 1995 and work begun at the NGMS International Conference, held in Irvine, California, USA, in February 1996. 
The goals of the NGMS IMS Project are to:

- Develop a unifying description of NGMS, an NGMS Specification that captures the results of the individual R\&D activities, and a framework for ensuring the integrability of the results into cost-effective NGMS.

- Develop on-line facilities for tracking and presenting advanced technologies and processes and advanced materials that will be used in and by NGMS, gauging their readiness for application.

- Develop an integratable set of models and simulations merging a bottoms-up view of the factory floor as it will be found in NGMEs with a top-down view of the globally distributed virtual enterprises.

The unique strength of the NGMS IMS effort is its systems approach. Starting with the NGME vision, the effort has adopted a needs-based understanding of the characteristics of future manufacturing systems and has defined an R\&D agenda to develop the best ideas on advanced manufacturing systems and integrate them into NGMS. The key issues of NGMS have to do as much with the integrability of manufacturing technologies and processes.

\section{WORK PACKAGES AND TASKS}

The Project objectives will be accomplished in a set of tasks clustered in three Work Packages.

\subsection{Work package 1}

This Work package will provide the framework for the NGMS IMS effort.

Task 1.1. Description of NGMS, will provide a standard description of NGMS, with key words defined and key concepts described, using the four central concepts, augmented with additional ideas on advanced manufacturing systems. Where different words are used to describe similar concepts, a mapping will provide a shared understanding of the vocabularies used to articulate the concepts.

Task 1.2. Specification of NGMS will maintain the NGMS Specification as a timely and complete documentation of the vision and functions of NGMS and as the definitive statement of the context in which the NGMS IMS Program's Work Packages and tasks will be pursued. The updated Specification will become a progressively more detailed description of NGMS as the results of a succession of Work Packages performed by the NGMS IMS effort are integrated into it.

Task 1.3. NGMS Systems Integration has two sub tasks. In the first, cross-Regional task team is developing and maintaining an NGMS systems integration framework, considering both horizontal integration (e.g. the things that relate to the floor level) and vertical, integrating functions at the floor, factory, and enterprise levels. The task team will identify inconsistencies and ambiguities among the Work Packages; where appropriate, it will recommend interface specifications for ensuring NGMS integrability. In the second sub task, the cross Regional task team will identify requirements for one or more systems integration testbeds where the 
integrability of NGMS IMS Program work products can be evaluated.

\subsection{Advanced Knowledge in Manufacturing Systems}

We will speed the application of advanced technologies, methodologies, systems, and materials by developing publicly accessible knowledge bases usable by process engineers. The intent is to package knowledge developed by the partners in the NGMS effort, by other IMS projects, and from other sources into an on-line system, called NGMnet. The knowledge bases will include a description of the innovative technology, methodology, system, or material, implementation and experience information, and an assessment of the risk of adoption. sign. A second sub-task will develop an interactive, on-line, Handbook of Standard Fixes. New technologies often are buggy, but be made reliable and useful when used in restricted ways or with the application of a small patch. The discovery of the fixes to the bugs can be a time-consuming process that is repeated as companies attempt to use the new technology. The Handbook will provide a vehicle for process engineers to record and propagate the fixes they discover and for other process engineers to access fixes as (or before) they encounter bugs.

\subsection{Work package 3}

There are four tasks involving modelling NGMS from four different perspectives. These four tasks will be conducted primarily as Regional tasks; a cross-Regional task will ensure that the tools used (e.g., object-oriented modelling tools) are consistent and that resultant models present a consistent representation of NGMS.

Task 3.1. Modelling and Simulation of Agile Manufacturing Systems is derived from on-going work in Autonomous and Distributed Manufacturing Systems (ADMS) being conducted under the Japanese Domestic IMS Program Task II-1. In this task we are developing

- Position of ADMS in NGMS

ADMS aims to fulfill the characteristics that are required in NGMS, such as flexibility, quick response, adaptability, globally, and concurrency. The system configuration of ADMS is autonomous and distributed, and its aim is cooperation and harmony.

Viewing ADMS from NGMS as a whole, it focuses mainly on the production phase among the life cycle, which includes development, design, production, physical distribution and post-sales, as shown in Fig 4. The subject of the research is modelling and operation in the phase. 


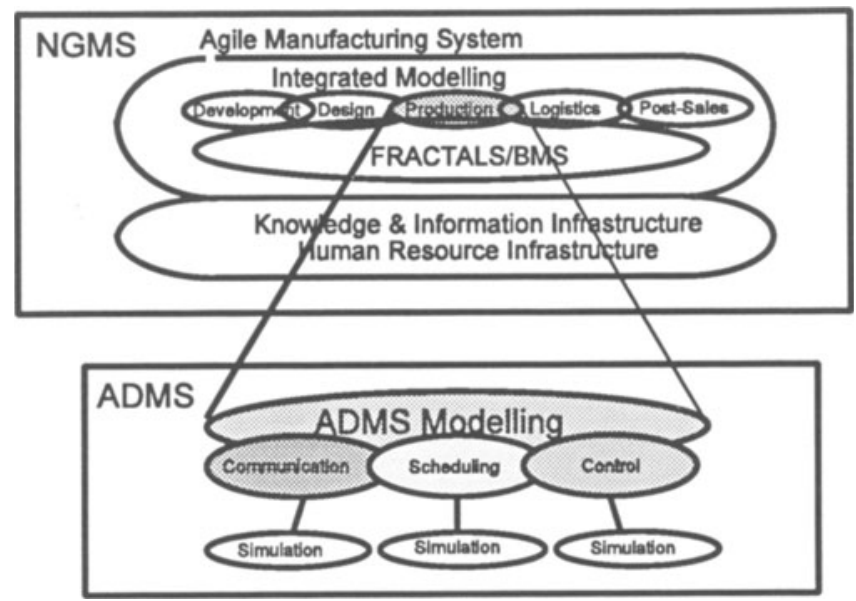

Figure 2

\section{- Modelling of ADMS}

Modelling provides the basis for the realization of ADMS, and it corresponds to the architecture of information processing. In the research on ADMS, we develop three kinds of modelling tools. They are STN(Scene Transition Net), Agent Net, and Job Model, as shown in Figure 5. Their common bases are the object-oriented technology, the discrete system theory, and the dynamic system theory.

STN is composed as a hybrid system that is able to integrate and deal with both continuous and discrete events. It aims to take in and integrate models that are based on even more different aspects, and to perform a wide range simulation of manufacturing system.

Agent Net aims to be applied in real-time control and scheduling, by merging Petri Net and object-oriented technology, and combining functions of cooperation, learning and self-organization.

Job Model aims to be applied in intelligent communication, which is to support computer-aided manufacturing in autonomous and distributed way. It attempts to take human factors into traditional product models and factory models. 


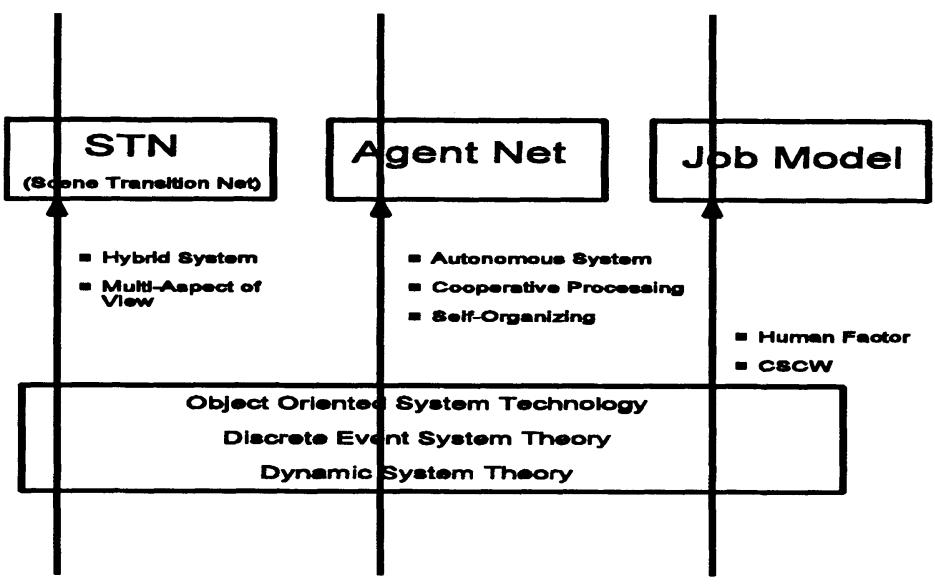

Figure 3

\section{- Operation of ADMS}

Operation refers to the decision support on manufacturing system, which is realized on the basis of modelling technology, and involves actions such as communication, control, and scheduling.

(a) Autonomous Distributed Scheduling

Development of a scheduling system which has the function to pursue self optimization in each individual process, and to simultaneously cooperate with other processes and aim for total harmonization.

(b) Autonomous Distributed Control

Development of control technology for autonomous distributed manufacturing system, which has robustness to troubles, flexibility, and easiness for construction, by using Agent Net.

(c) Intelligent Communication

Development of intelligent ways of communication to realise HIM (Human Integrated Manufacturing), which is an advanced form of CIM that harmonizes machines and humans, by using Job Model.

Task 3.2. Next Generation Enterprise Modelling, Simulation, and Operations will develop new forms of enterprise models that will provide assistance in the formation, transition and management of NGMEs. The task will focus on the relationships and communications between individual autonomous work units when they are either participating in a single enterprise or participating simultaneously in several virtual enterprises. This task will focus on identifying the right partners and the right interfaces among partners in an NGME.

Task 3.3. Modelling for Biological Manufacturing Systems will establish basic models for Biological Manufacturing System (BMS). It is well-known that for NGMS, a manufacturing 
system with autonomous distributed function is required. However, it is not always the right way to construct it as an extension of traditional method. The methodology of system construction is an important point, for it is inevitable for NGMS to be in harmony with society and nature.

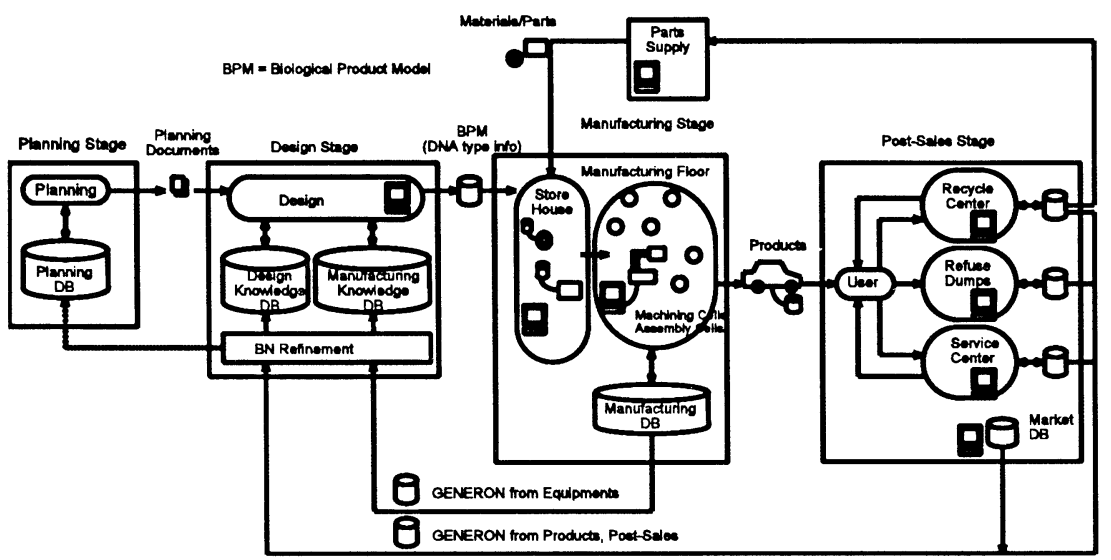

Figure 4

BMS is 'a manufacturing system learning from and living with biological life'. By introducing into artifacts the excellent ability of biological life, such as self recognition, self growth, self recovery, evolution and adaptation, BMS conceives interactions between human beings and artifacts, and furthermore, tries to locate artificial system in the macro ecosystem, covering the whole life cycle of a product, i.e. planning, design, production, operation, maintenance, recycling and disposal.

Figure 6 shows the outline of the stage configuration and the information flow of BMS.

Figure 7 shows the entire view of the research on BMS. Considering the life cycle, its topics can be listed up as follows:

\section{(1) BMS Core System}

Research mainly based on the way to construct a Biological Product Model(BPM), which is a key to BMS, in an attempt to obtain a basic mechanism by which biological characteristics are applied.

(2) DNA-Oriented Design System

Research on evaluating-type design by using biological product model.

(3) Biological Information Processing Function

Research on information processing function that biological facilities are supposed to have, focusing mainly on production stage. 
(4) Product Life Cycle Feedback

Research on heredity and evolution of a product.

(5) Harmonization with Macro Ecosystem

Research on total life cycle including disposal and recycling, by using simulation, etc.

Although the above research topics are closely related with one another, we intend to concentrate mainly on the topics (1), (2), and (3)for the time being.

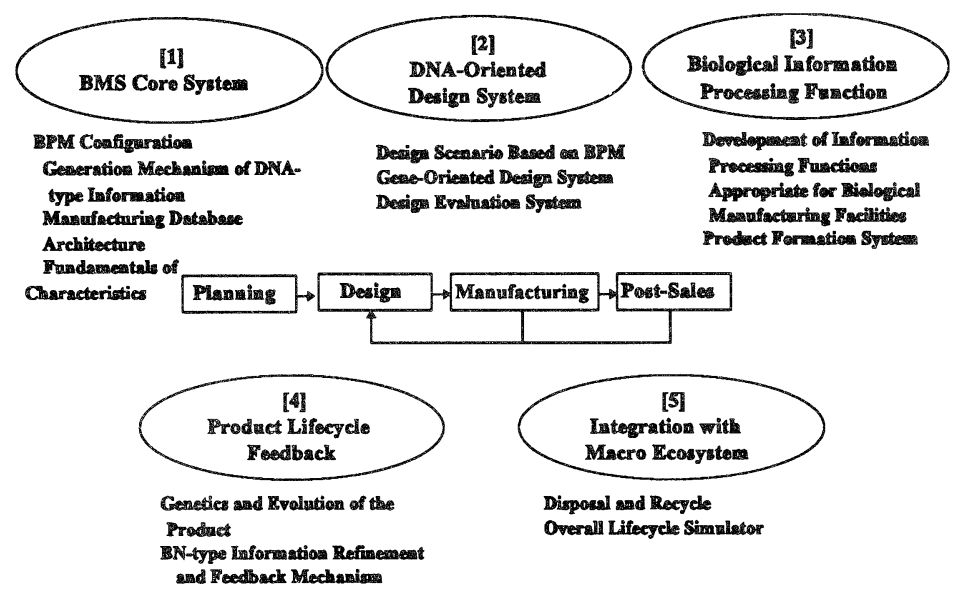

Figure 5

Task 3.4. Modelling for Virtual Enterprise will develop models to help in the formation and management of virtual enterprises, considering both the enterprise as an entity and individual work units that may be participating in several virtual enterprises. The models will illuminate the decision points in the enterprise life cycle; e.g. to illuminate the decision to combine to offer a product at a competitive target price and to assist individual autonomous work units in their decisions to commit to participation in multiple enterprises.

Task 3.5. Modelling Tools and Model Integration will establish the mechanism to integrate the four modelling tasks. There are two major integration sub-tasks: tools and models. Each assumes that the modelling tools will be based on the object-oriented programming paradigm and each has object oriented tools under development. An objective of this task is to ensure that the tools are compatible, that their semantics and interfaces are consistent. A set of NGMS IMS Program standards, that will inform the establishment of international standards and conform to them once established, will be developed by a cross Regional task team. Each of the modelling tasks assumes that work units will conduct negotiations as they cooperatively reach decisions relating to the enterprise's goals and their individual roles in meeting those goals. A cross Regional task team will find the commonalties among the algorithms and methodologies used in the other tasks to find optimizations and to help establish standards. 
As the four concepts of Agile Manufacturing, Autonomous and Distributed Manufacturing System, Biological Manufacturing System, and Fractal Company mature, each will contribute toward a unified view of NGMS. Our assessment of the way these concepts will mature over time is shown in Figure 8. At any given time, the next generations of manufacturing systems will be a combination of the most useful ideas coming from the four concepts. The combination will change over the lifetime of the NGMS IMS Program.

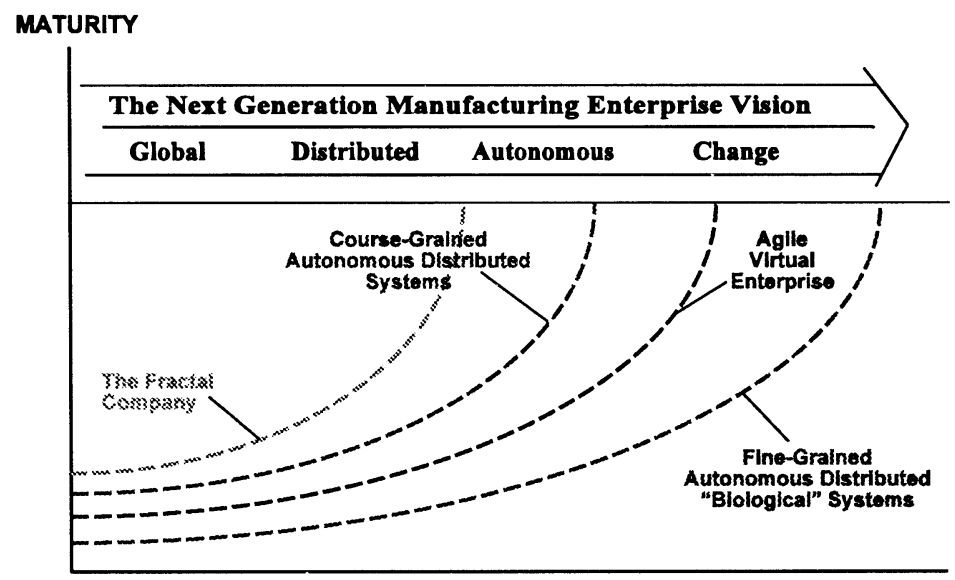

TIME

Figure 6

7 OPERATION AND MANAGEMENT STRUCTURE FOR THE INTERNATIONAL COOPERATIVE RESEARCH

The NGMS IMS Program is organized as follows:

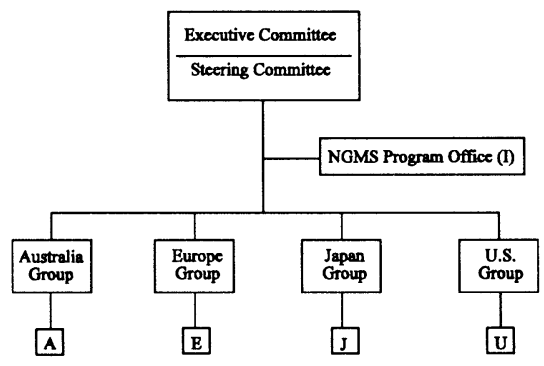

Figure 7

Coordinating Partner I. International:

A. Australian Group:

E. European Group:

J. Japanese Group:

U. The Group in the USA:
CAM-I NGMS Program Office Expected to join in the near future CAM-I European Office Fuji Electric Co., Ltd. CAM-I NGMS Program Office 
The administrative management of this IMS Project is the responsibility of the NGMS IMS Program Office, as international coordinator, and the Regional Coordinating Partners. The Program Office is responsible for coordinating the inter Group aspects of the Program and will be the Program's liaison with the International IMS Steering Committee and with the International IMS Secretariat.

\section{SUMMARY}

The first results from the NGMS R\&D program will be available in 1997; others will flow in the next two years. Taken together, the results will lead to a transformation of the NGMS partners' manufacturing systems into those that can support fast moving global enterprises in rapidly changing and very competitive markets.

\section{ACKNOWLEDGMENTS}

The requirements of Next Generation Manufacturing Systems are a synthesis of contributions made in NGMS Program workshops and conferences by the NGMS project partners and other thought leaders from many manufacturing companies and universities. 